\title{
Repeat Knot Formation in a Patient with an Indwelling Ureteral Stent
}

\author{
Brian Eisner, Howard Kim, Dianne Sacco \\ Department of Urology, Massachusetts General Hospital and Harvard Medical School, Boston, \\ Massachusetts, USA
}

\begin{abstract}
A patient treated for nephrolithiasis formed knots in 2 occasions, in 2 separate indwelling ureteral stents. This rare complication may make stent removal difficult. To our knowledge, this is the first case report of repeat knot formation in a single patient.
\end{abstract}

Key words: ureter; stent; complications; lithiasis

Int Braz. J Urol. 2006; 32: 308-9

\section{INTRODUCTION}

A rare complication of indwelling ureteral stents is knot-formation within the body or coiled portion of the ureteral stent. Several techniques for removal have been described, including simple traction, removal with guide wire assistance, ureteroscopy, and percutaneous removal (1-3). We report a case in which a patient formed knots in 2 separate stents.

\section{CASE REPORT}

An 82-year-old critically ill female with multiple medical problems, including multiple sclerosis requiring tracheostomy, was treated for an $11 \times 8 \mathrm{~mm}$ left renal stone with a Cook Kwart Retro-Inject stent $(6 \mathrm{~F} \times 22-32 \mathrm{~cm})$ in August, 2002. She was lost to follow up until July 2003 when she developed Escherichia coli urosepsis. The stent was thickly encrusted requiring 2 shock wave lithotripsies and a ureteroscopy with laser lithotripsy to facilitate re- moval. Examination revealed a knot in the proximal coiled portion. Due to extensive manipulation during the procedure, a new Kwart stent was placed with the plan to remove it within a short period of time.

An attempt to remove the stent using an externalized string was unsuccessful in the clinic. Radiography revealed a knot in the proximal coiled portion of the stent (Figure-1). The patient was taken to the operating room for planned cystoscopy with possible ureteroscopy to perform complex stent removal. During attempted induction of anesthesia, difficulties with the patient's tracheostomy caused several forceful coughs, producing a Valsalva effect. Due to the difficulty of anesthesia and complexity of the patient, prior to instrumentation, the stent was easily removed by simple traction. The knot was no longer present.

\section{COMMENTS}

Knot formation is a rare complication of indwelling ureteral stents. We report a patient who 


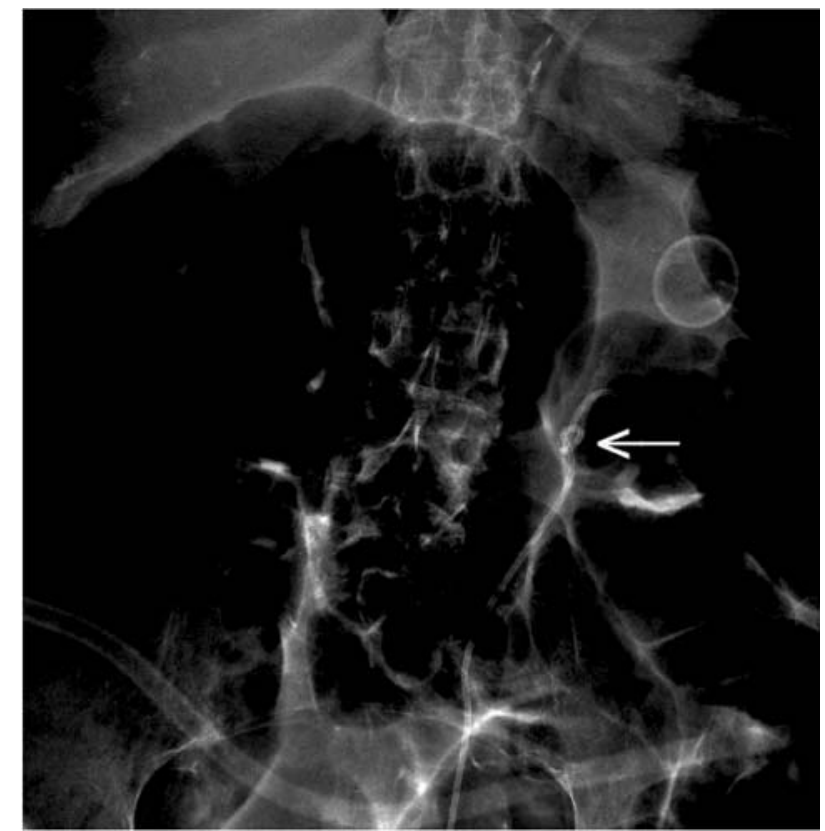

Figure 1 - Knot formation seen on abdominal X-ray (arrow).

formed knots in 2 indwelling stents. Previous authors have hypothesized that stent length, as well as coil configuration may be contributing factors to stent knot formation (3). We surmise that the anatomy of the patient's renal pelvis in conjunction with the use of a Cook Kwart Retro-Inject stent with multiple proximal coils increased the likelihood of knot formation.

Several authors have described novel methods to remove knotted stents (1-3). To our knowledge, this is the first report of repeat stent knot formation on a single patient. In our patient, the first stent required ureteroscopic treatment of encrusted stone fragments for removal. The second knotted stent was in place for a short period without evidence of encrustations. Repeated Valsalva (coughing) was the only interim event that could account for the knot uncoiling. We hypothesize that increase in intraabdominal pressure caused movements in the position of the kidney, ureter, and/or indwelling stent, causing the knot to come undone. Valsalva is an easy and relatively innocuous treatment that may aid in removal of knotted stents and avoidance of more invasive procedures.

\section{CONFLICT OF INTEREST}

None declared.

\section{REFERENCES}

1. Kundargi P, Bansal M, Pattnaik PK: Knotted upper end: a new complication in the use of an indwelling ureteral stent. J Urol. 1994; 151: 995-6.

2. Baldwin DD, Juriansz GJ, Stewart S, Hadley R: Knotted ureteral stent: a minimally invasive technique for removal. J Urol. 1998; 159: 2065-6.

3. Quek ML, Dunn MD: Knot formation at the mid portion of an indwelling ureteral stent. J Urol. 2002; 168: 1497.

\footnotetext{
Correspondence address:

Dr. Brian Eisner

Department of Urology

Massachusetts General Hospital

55 Fruit Street

Boston, Massachusetts, 02114, USA

Fax: +1 617 726-6131

E-mail: beisner@partners.org
} 